Humanités numériques

\title{
Pietro Liuzzo, Digital Approaches to Ethiopian and
} Eritrean Studies

Wiesbaden, Harrassowitz Verlag, 2019

\section{Anaïs Wion}

\section{OpenEdition}

\section{Journals}

Édition électronique

URL : https://journals.openedition.org/revuehn/2314

DOI : 10.4000/revuehn.2314

ISSN : 2736-2337

Éditeur

Humanistica

\section{Référence électronique}

Anaiis Wion, « Pietro Liuzzo, Digital Approaches to Ethiopian and Eritrean Studies », Humanités numériques [En ligne], 4 | 2021, mis en ligne le 01 décembre 2021, consulté le 01 décembre 2022. URL : http:// journals.openedition.org/revuehn/2314 ; DOI : https://doi.org/10.4000/revuehn.2314

\section{(c)}

Creative Commons - Attribution 4.0 International - CC BY 4.0 https://creativecommons.org/licenses/by/4.0/ 


\section{humanités \\ numériques}

$4 \quad 2021$

Varia

COMPTE RENDU

\section{Pietro Liuzzo, Digital Approaches to Ethiopian and Eritrean Studies}

Wiesbaden, Harrassowitz Verlag, 2019

\section{Anaïs Wion}

Référence(s) :

Pietro Liuzzo, 2019. Digital Approaches to Ethiopian and Eritrean Studies. Wiesbaden : Harrassowitz Verlag. 280 p.

\section{Entrées d'index}

MOTS-CLÉS : littérature, épigraphie, manuscrit, TEI, Web sémantique

KEYWORDS: literature, epigraphy, manuscript, TEI, semantic Web

Cet ouvrage présente les travaux menés à l'université de Hambourg au Hiob Ludolf Center for Ethiopian and Eritrean Studies (HLCEES) autour du projet Beta Mașāḥıft (BM) en donnant accès aux réflexions et aux choix scientifiques et techniques présidant à la création d'un environnement numérique pour les études sur et à partir des textes éthiopiens classiques ${ }^{1}$. Bien au-delà de ce périmètre aréal, Digital Approaches to Ethiopian and Eritrean Studies peut être lu comme une présentation extrêmement pédagogique de ce que représentent désormais les données numériques dans les sciences humaines et sociales. Fondé sur de nombreux exemples pratiques et sur une solide expérience, l'ouvrage propose une ample réflexion théorique sur l'ouverture des données et sur ce que peut être une réelle interopérabilité. Il donne à comprendre ce que les " humanités numériques " apportent au monde de la recherche et comment les nombreux outils de description, d'interrogation, de manipulation, de visualisation des sources offrent un nouveau paradigme 
scientifique, celui des Linked Open Data (LOD ou «données ouvertes liées ", plus connues en français sous l'appellation "Web sémantique ") et de l'« histoire faite à partir des données " (Data Driven History).

Après un état de la question sur les usages des outils numériques dans les études éthiopiennes depuis les années 1990 avec les remarques pionnières de Manfred Kropp (1994) et les premières campagnes de numérisation, le premier chapitre expose comment des données produites dans le cadre du projet BM peuvent être réutilisées, par exemple en étant exportées et visualisées par d'autres applications. L'objectif de ce chapitre est de démontrer que la structuration des données en XML-TEI permet de créer un environnement de travail stable, facilement accessible, que chacun et chacune peut réemployer à sa guise. C'est notamment parce qu'il s'agit de mettre en œuvre toute la souplesse de l'encodage en TEI que le projet BM a choisi de ne pas saisir les données dans un formulaire et qu'il enjoint les participants et participantes à comprendre la logique d'un fichier TEI (régulé par un schéma ODD qui limite les balises utilisées et contrôle leur usage) et à écrire directement dans un éditeur.

Le chapitre 2 confronte des données encodées dans le projet BM à des données existantes dans d'autres projets et tente de les faire dialoguer. Ce «crash test " comparatif est effectué à partir de corpus épigraphiques. L'épigraphie grecque est la spécialité d'origine de l'auteur, qui a obtenu un doctorat en histoire antique avant même d'être ingénieur en informatique. Sa page GitHub annonce en outre avec humour "I never knew I could do this ». En alignant les encodages sur le standard EAGLE pour l'épigraphie antique ${ }^{2}$ - qu'il a participé à établir -, il examine les différents modèles de description des inscriptions et la définition même d' « inscription épigraphique ». La discussion sur les liens entre la finesse de l'encodage et l'appréhension de ce qu'est une inscription épigraphique montre la puissance désignatoire que peut avoir un encodage, si un travail prudent de compréhension des normes descriptives, toujours différentes suivant les traditions académiques, n'est pas d'abord effectué. Pour le dire plus simplement, le choix des balises TEI peut enfermer l'interprétation des textes dans des catégories : aussi leur usage et leur compréhension doivent-ils être soigneusement définis et compris.

Dans cette même perspective, le chapitre 3 commence par étudier la définition d'un texte manuscrit et l'implication de cette définition dans la structuration des données, lorsqu'il s'agit de cataloguer un témoin matériel, de reconnaître des œuvres littéraires ou de rendre compte des transformations des artefacts écrits. Pour cela, la philosophie du projet $\mathrm{BM}$ se nourrit de nombreuses références théoriques. Parmi celles-ci, La Syntaxe du codex de Andrist, Canard et Maniaci (2013) et les travaux dirigés par Orlandi pour l'établissement de la Clavis Coptica ${ }^{3}$ jouent un rôle fondamental, respectivement pour la compréhension des éléments matériels et pour la définition des œuvres littéraires.

À nouveau, Pietro Liuzzo déploie un exemple d'utilisation des données, montrant comment des visualisations en diagrammes de flux font apparaître, sous une forme tout à fait innovante, les évolutions de la littérature éthiopienne (à partir des données encodées jusqu'à présent, et donc des limites explicitées de ce jeu de données). Ces exemples n'ont pas vocation à démonstration mais permettent aux lecteurs de se figurer comment les données peuvent être transformées et interrogées. C'est l'un des chevaux de bataille de cet ouvrage que d'appeler son lectorat à 
exercer à la fois son autonomie et sa créativité et à se saisir pleinement des outils à sa portée pour répondre à des questionnements scientifiques. Si le lien entre visualisation des données et analyse précède largement l'ère numérique, il n'en demeure pas moins que peu nombreux sont les praticiens des sciences humaines et sociales qui maîtrisent cette sémiologie. Là encore, c'est tout un enseignement qui est nécessaire pour acquérir ces compétences, conceptuelles autant que pratiques.

Le chapitre 4 fait la part belle à la géographie historique - en commençant par une présentation rapide de la nécessaire différenciation entre un toponyme, une localisation et un lieu, telle que formalisée dans Pleiades notamment ${ }^{4}$. Le hiatus entre ces trois points d'entrée est crucial pour faire état de la dynamique de la géographie historique et de ses incertitudes. Se fondant sur un exemple, P. Liuzzo choisit de comparer les termes utilisés dans l'Antiquité tardive (en guèze et en grec) pour désigner le royaume chrétien de la Corne (Aethiopia, Hbst, Aksum, Ityopia) et dévoile comment le codage de chacun de ces termes, associés à leurs provenances, à leurs dates d'usage, aux sources qui les emploient et aux territoires qu'ils désignent, permet de réaliser une analyse comparative complexe. En ajoutant des relations entre les termes, les concomitances et les césures peuvent être appréhendées (avec un exemple de visualisation dans Peripleo, un moteur de recherche et un outil de visualisation de données spatiotemporelles).

Ce chapitre montre également comment l'usage d'algorithmes de geoparsing permet de traiter rapidement de grands corpus, puis explicite les liens entre l'encodage de l'élément < placeName > et le géoréférencement, de manière très pratique. Comme dans les autres chapitres, la conclusion insiste sur la nécessité d'un effort collectif pour renseigner ces données en se saisissant des outils à disposition, afin que la communauté scientifique dispose d'ici quelques années de données ouvertes et interopérables, qui aident à comprendre la mutabilité des lieux et des territoires, leurs continuités et discontinuités, à penser les itinéraires et les routes ou encore les déplacements de populations. Cette diversité de l'appréhension des espaces peut être modélisée si l'encodage en offre la possibilité, et là encore l'auteur lance un appel pour que les ressources numériques soient utilisées avec la conscience de toutes leurs potentialités.

Le chapitre 5 revient sur la pierre angulaire du projet BM que furent la numérisation et l'outillage du dictionnaire guèze-latin d'August Dillmann (1865). La langue guèze est une langue éthio-sémitique ancienne qui fut l'unique langue écrite de l'Éthiopie chrétienne depuis l'Antiquité tardive jusqu'à la période moderne. Ce dictionnaire fut, dès l'année 2000 à l'université de Naples, transformé en un fichier TXT, puis encodé, lemmatisé et enfin relié aux textes bibliques transcrits et encodés dans le projet BM. Ce Lexicon ${ }^{5}$ virtuel est corrigé et enrichi en permanence.

Le chapitre 6 présente le volet parallèle au dictionnaire numérique, à savoir un projet de reconnaissance automatique de la grammaire guèze (morphological parser), développé en premier lieu par le projet européen TraCES entre 2014 et $2019^{6}$. Très technique, ce chapitre explique, pas à pas, comment se conçoit l'automatisation du guèze, sans cacher les limites actuelles de cet outil. Le réemploi des données structurées est une 
fois de plus illustré, ici en montrant comment Alpheios ${ }^{7}$ - projet de développement d'outils open source pour l'étude des langues anciennes peut permettre de visualiser ces données.

À ce stade, l'ouvrage a mis en avant chacune des facettes ou des briques qui constituent à ce jour l'environnement de travail du projet BM. Le chapitre 7 explore alors plus avant la notion de Linked Open Data (LOD) et son lien avec les ontologies. Bien que des passages puissent rester sibyllins, l'essentiel du texte demeure accessible. P. Liuzzo y affirme que l'interopérabilité entre des environnements de recherche n'est pleinement fonctionnelle qu'à condition de comprendre comment opérer des ponts entre les jeux de données et il en explicite les tenants et les aboutissants. Différence et complémentarité entre données descriptives et données relationnelles sont clairement expliquées.

Le chapitre 8 concerne l'édition numérique et s'attache avant tout à préciser les transformations d'un fichier XML. Il montre qu'une édition numérique, aussi connectée soit-elle à d'autres systèmes, peut tout simplement se traduire par une édition en HTML ou en PDF.

Le chapitre conclusif ouvre une réflexion sur l'ouverture des données. À la façon parfois drolatique, poétique ou philosophique de ce livre nourri de nombreuses références, c'est Italo Calvino qui est convoqué ici pour cette apologie de l'" openness ", mais aussi de la "multiplicité " et de l'« exactitude ». Il en est des architectures numériques comme des «villes invisibles ": elles sont ramifiées, jointoyées, connectées, non hiérarchiques, mais surtout les unes comme les autres regardent de chaque côté du temps, vers le passé comme vers le futur.

Cet ouvrage dense est un véritable plaidoyer pour une philosophie ouverte et collaborative des environnements numériques et un appel à la créativité dans la manipulation des données, cette créativité des questions de recherche n'étant récompensée que si les données sont solidement construites, bien entendu. Cela nous engage alors à poser la question suivante : à quel niveau de la critique des sources se situe cette proposition, qui nous est faite collectivement et technologiquement, d'opérer un changement de paradigme pour faire de la Data Driven History ? Et concrètement, quelles formations doit-on mettre en place - en France, en particulier - pour se saisir de ces outils conceptuels et technologiques?

Le projet BM, dans sa précision comme dans son gigantisme, est rendu possible par l'accumulation de financements sur projets, le plus important - sur lequel repose la possibilité même d'un tel projet - étant garanti pendant quatre décennies par la Deutsche Forschungsgemeinschaft (DFG), institution chargée de promouvoir et de financer la recherche scientifique en Allemagne. Dans cette enveloppe, aucun poste fixe n'est prévu et il s'agit donc d'une recherche institutionnellement garantie à long terme mais reposant humainement uniquement sur des contractualisations. L'architecture et le modèle économique sont donc bien différents de ce que nous tentons de préserver de notre côté du Rhin. Et pourtant, la force opératoire et l'attractivité de ce projet sont indéniables. C'est notamment grâce à cet empilement de financements et de contrats qu'est possible le travail d'encodage directement en TEI, puisque les participantes et participants au projet BM sont pris en charge et suivis au sein de l'équipe du Hiob Ludolf Center de l'université de Hambourg. Au-delà de ce noyau très dynamique et malgré les nom- 
breux ateliers et le réel talent pédagogique des animateurs et animatrices du projet, la prise en main de cet environnement par des contributeurs ou contributrices externes reste un frein au travail collectif. Les guidelines ne suffisent pas pour se repérer dans les différentes facettes d'un workflow, certes classique (usage de GitHub et compréhension du XML) mais mal maîtrisé par la plupart des chercheurs et chercheuses en sciences humaines et sociales - à tout le moins aujourd'hui et en France.

\section{Bibliographie}

Andrist, Patrick, Paul Canart et Marilena Maniaci. 2013. La Syntaxe du codex. Essai de codicologie structurale. Turnhout : Brepols.

Dillmann, August. 1865. Lexicon linguae aethiopicae. Cum indice latino. Adiectum est vocabularium tigre dialecti septentrionalis compilatum a Werner Munziger. Lipsiae : T. O. Weigel. ht tps://archive.org/details/lexiconlinguaeae00dilluoft.

Kropp, Manfred. 1994. «From Manuscripts to the Computer : Ethiopic Studies in the Last 150 Years ». Dans The Edward Hincks Bicentenary Lectures, édité par Kevin J. Cathcart, 114137. Dublin : Department of Near Eastern Languages, University College Dublin.

Orlandi, Tito. s. d. « Clavis Patrum Copticorum. An Index of the Authors and Works of the Coptic Literature ». http://www.cmcl.it/ cmcl/chiam_clavis.html.

\section{Notes}

1 Voir https://betamasaheft.eu. De nombreuses données et ressources de l'ouvrage sont disponibles en ligne : https://pietroliuzzo.github.io/DHEth/.

2 EAGLE, pour Europeana Network of Ancient Greek and Latin Epigraphy. Voir https://w ww.eagle-network.eu pour les informations relatives à ce projet fondamental.

3 http://www.cmcl.it/ cmcl/chiam_clavis.html.

4 Pleiades est un gazetier de toponymes et lieux antiques. Voir https://pleiades.stoa.org.

5 https://betamasaheft.eu/Dillmann/.

6 From Translation to Creation : Changes in Ethiopic Style and Lexicon from Late Antiquity to the Middle Ages, https://www.traces.uni-hamburg.de.

7 https://alpheios.net.

\section{Auteur}

\section{Anaïs Wion}

UMR 8171 IMAF, CNRS, Aubervilliers, France

ORCID 0000-0001-6568-9257

anais.wion@univ-paris1.fr

\section{Droits d'auteur}

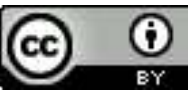

Les contenus de la revue Humanités numériques sont mis à disposition selon les termes de la Licence Creative Commons Attribution 4.0 International. 